\title{
Application of autologous stem cell transplantation in various adult and pediatric rheumatic diseases
}

\author{
Alan Tyndall ${ }^{1}$
}

In the past 15 years, more than 1,500 patients worldwide have received a hematopoietic stem cell transplant, mostly autologous, as treatment for a severe autoimmune disease (AD). A recent retrospective analysis of 900 patients showed that the majority had multiple sclerosis, systemic sclerosis, systemic lupus erythematosus (SLE), rheumatoid arthritis (RA), and juvenile idiopathic arthritis (JIA; $n=65$ ) and idiopathic cytopenic purpura. An overall 85\% 5-year survival and 43\% progression-free survival was seen, with 100-day transplant-related mortality (TRM) ranging between 1\% (RA) and 11\% (SLE and $J \mid A)$. Around $30 \%$ of patients in all disease subgroups had a complete response, despite full immune reconstitution. In many patients, morphological improvement was documented beyond any predicted known effects of intense immunosuppression alone. It is hoped that the results of three ongoing large prospective, randomized, controlled trials will allow modification of the protocols to reduce the high TRM, which relates to regimen intensity, age of patient, and comorbidity. Multipotent mesenchymal stromal cells (MSCs), including autologous MSCs, have recently been tested in various ADs, exploiting their immune-modulating properties and apparent low acute toxicity. Despite encouraging small phase I/II studies, no positive data from randomized, prospective studies are as yet available in the peer-reviewed literature.

\section{HEMATOPOIETIC STEM CELL TRANSPLANTATION IN AUTOIMMUNE DISEASE}

In 1996, an international collaboration began to explore the concept of immune ablation, or at least the major reduction of autoaggressive cells, in patients suffering from severe autoimmune disease $(\mathrm{AD})$ and not responding to conventional therapy (1). It was hoped that following reconstitution of the immune system, a "resetting" of the autoimmune process would occur. The idea arose from coincidental case reportshematopoietic stem cell transplant (HSCT) given for the treatment of malignancy with coexisting AD (2) - and was corroborated by animal model data (3). The first patients receiving an HSCT for an AD had systemic sclerosis (SSc) $(4,5)$ and responded satisfactorily with durable remissions.

From the outset, an international collaboration ensued that is committed to establishing the role, if any, of HSCT in the treatment of severe therapy-resistant $\mathrm{AD}$ in the context of prospective, randomized clinical trials coupled with mechanistic side studies (6).

Although many protocols were employed, they basically ranged from less aggressive (e.g., $200 \mathrm{mg} / \mathrm{kg}$ cyclophosphamide (CYC) plus antithymocyte globulin (ATG)) to more intensive (e.g., total body irradiation (TBI) plus CYC/ATG and CD34 selection).

Up to one-third of the patients in all groups experienced a significant clinical improvement, including full and drugfree sustained remissions (7). In some of those studied (systemic lupus erythematosus (SLE), multiple sclerosis (MS), and SSc), remission was sustained despite full immune reconstitution. In SLE, the authors demonstrated that the humoral responses to recall antigens (tetanus, polio, measles, and mumps) were ablated following autologous HSCT, as expected, but in addition, eradication of autoantibodies such as anti-double-stranded DNA was also achieved coincident with clinical remission in five cases (8). Following immune reconstitution, a comparison of the T-cell receptor $\mathrm{V} \beta$ repertoires between the patients and normal subjects showed a completely normal pattern. Despite this, no patient had relapsed in the 8-year follow-up reported. Similar findings were described in seven MS patients who remained in remission up to 3 years post-transplant despite regaining a normal T-cell repertoire (9).

\section{AUTOLOGOUS VS. ALLOGENEIC HSCT}

The above findings are particularly important because at the onset of the project many considered autologous HSCT to be doomed to failure, given that the identical "autoaggressive" immune system was being given back to the patient. However, the initial choice of autologous over allogeneic HSCT was mainly based on the lower toxicity of autologous HSCT, mainly because of graft-vs.-host disease associated with allogeneic HSCT. It is now appreciated that in many patients who achieved clinical remission, the autoaggressive immune system was "debulked" rather than fully ablated, allowing reestablishment 
of normal immune regulation, in part because of increased regulatory $\mathrm{T}$ cell (Treg) numbers and activity (10).

\section{MORE THAN JUST PROLONGED IMMUNOSUPPRESSION}

Several groups have published findings of reduced collagen deposition in skin (11) and normalization of microvasculature in SSc patients $(12,13)$ following autologous HSCT (Figure 1). None of these observations is readily explained by either sustained immunosuppression or direct effects on fibroblasts and

a

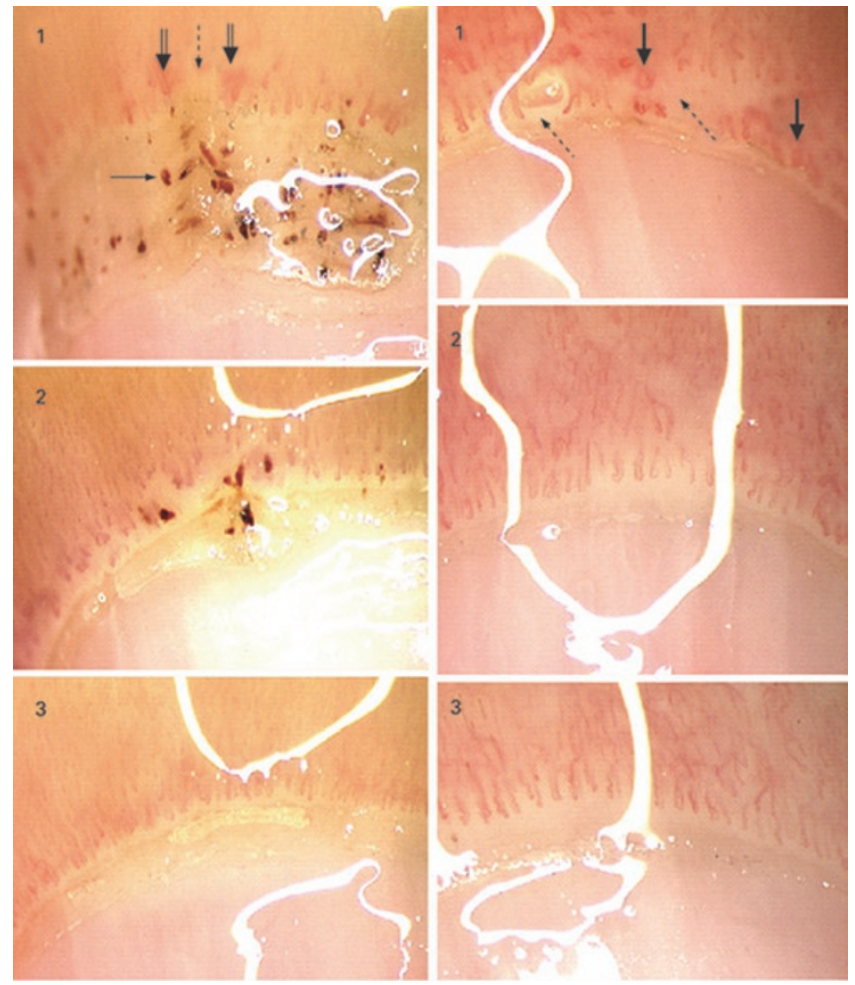

Figure 1. Normalization of microcapillaries after hematopoietic stem cell transplant (HSCT) in two patients with (a) systemic sclerosis (SSc) and (b) mixed connective tissue disease (MCTD) (1) pre-HSCT, (2) $1 \mathrm{mo}$, and (3) 3 mo post-HSCT. Large arrows show microhemorrhages and small arrows indicate loss of capillaries (rarification).(Reprinted with permission from ref. 38. Copyright ( 2008 BMJ Publishing Group and European League Against Rheumatism.) endothelial cells, and they suggest a more profound modulation of the inflammatory niche by mechanisms yet to be fully elucidated (12).

It is imperative to demonstrate through large randomized, prospective clinical trials the true impact of HSCT in AD. Currently, three such trials are running; ASTIS (Autologous Stem Cell Transplantation International Scleroderma), SCOT (Scleroderma Cyclophosphamide or Transplant), and ASTIC (Autologous Stem Cell Transplantation for Crohn's Disease). Details are available on their respective websites, and Table 1 includes a comparison and contrast. ASTIS and SCOT are similar in patient selection, control arms, and end points, but the transplant protocols differ. SCOT employs a more intense regimen including TBI. Each has experienced its own toxicity issues, all of which were previously known in HSCT medicine. Only time will tell which approach, if any, imparts a clinically useful and durable outcome; ASTIS has finished recruitment (156 patients) and the first efficacy analysis will be available early in 2012, with the last patient having reached the primary 2-year end point (how many alive with no end-stage organ failure) in October 2011. SCOT completed the recruitment of 75 patients in May 2011 and ASTIC is nearing its target of 40 patients (14).

A recently published smaller phase II randomized trial in SSc showed a positive outcome in the 10 transplanted patients compared with 9 control patients who received $1 \mathrm{~g} / \mathrm{m}^{2}$ CYC for $6 \mathrm{mo}$ (15). The conditioning regimen was $200 \mathrm{mg} / \mathrm{kg}$ CYC plus five doses of rabbit ATG, each accompanied by a 1-g intravenous infusion (IVI) of methylprednisone. The primary end point at 12 mo was based on a percentage improvement of the modified Rodnan skin score and/or pulmonary status. Although the low toxicity and positive outcome were gratifying, the low numbers of patients and short follow-up would currently caution against recommending HSCT as "standard of care" (16). In the ASTIS study, the first transplant-related mortality (TRM) occurred only after 54 patients had been randomized (17) and will be much higher than the estimated 6\% from the retrospective studies in the final analysis.

Since the initiation of the project, several events have radically changed the environment of AD treatment; the first biologics (anti-tumor necrosis factor- $\alpha$ (TNF- $\alpha$ ) monoclonal

Table 1. Large randomized clinical trials of autologous HSCT in autoimmune disease

\begin{tabular}{llll}
\hline & ASTIS & SCOT & ASTIC \\
\hline Principle investigator & J van Laar, UK & K Sullivan, USA & C Hawkey, UK \\
Target & 156 patients & 75 patients & 40 patients \\
Transplant regimen & $200 \mathrm{mg} / \mathrm{kg} \mathrm{CYC}$ & $120 \mathrm{mg} / \mathrm{kg} \mathrm{CYC}$ & $200 \mathrm{mg} / \mathrm{kg} \mathrm{CYC}$ \\
& $7.5 \mathrm{mg} / \mathrm{kg} \mathrm{ATG}$ (rabbit) & $90 \mathrm{mg} / \mathrm{kg} \mathrm{ATG}$ (equine) TBI $800 \mathrm{cGY}$ & $7.5 \mathrm{mg} / \mathrm{kg} \mathrm{ATG}$ \\
& CD34 selection & CD34 selection & Unselected graft \\
Control arm & Monthly CYC, & Monthly CYC, & Mobilization and then \\
& $750 \mathrm{mg} / \mathrm{m}^{2}$ IVI $\times 12$ & $750 \mathrm{mg} / \mathrm{m}^{2}$ IVI $\times 12$ & delayed transplant for 12 mo \\
Primary end point & Alive at 2 y without end-stage organ failure & Composite end point (death, end-organ & Proportion of patients in \\
& & failure) at 54 mo & sustained remission at 1y \\
Current status & Finalized_last patient randomized & Recruitment ended May 2011 & 37 of 40 patients randomized
\end{tabular}

ASTIC, Autologous Stem Cell Transplantation for Crohn's Disease; ASTIS, Autologous Stem Cell Transplantation International Scleroderma ; ATG, anti-thymocyte globulin;

CYC, cyclophosphamide; HSCT, hematopoietic stem cell transplant; IVI, intravenous infusion; SCOT, Scleroderma Cyclophosphamide or Transplant; TBI = total body irradiation. 
antibodies) reduced the need for more toxic therapies such as HSCT in rheumatoid arthritis (RA), and this was closely followed by similar developments in other AD treatment (e.g., natalizumab for MS, anakinra, canakinumab, and tocilizumab for juvenile idiopathic arthritis (JIA), and recently belimumab for SLE). However, none of these agents has induced sustained drug-free remission of $\mathrm{AD}$ and no such effective disease-modifying agent is available for SSc.

\section{TREATMENT-RELATED MORTALITY}

A major issue for physicians dealing with $\mathrm{AD}$ was and still is that patients rarely die immediately from their disease. However, a growing body of literature has suggested that uncontrolled systemic inflammation does lead to premature atherosclerosis and cardiovascular deaths (18), as well as toxicity from chronic immunosuppression, especially glucocorticoids. Despite this, it is still a challenge for a rheumatologist, neurologist, or gastroenterologist to accept an immediate TRM of 5-10\%, especially because long-term benefits have yet to be demonstrated. The hypothesis is that in a randomized, prospective trial of HSCT vs. conventional treatment, early toxicity from TRM would eventually be surpassed by later deaths and/or organ failure from disease progression in the control arm. This has yet to be proven.

Apart from the well-known acute toxicity of HSCT (infection and bleeding during the aplastic period and late infection during the T-cell reconstitution phase), several other factors emerged during the program. Some SSc patients experienced serious lung toxicity from TBI, whereas other SSc patients suffered a scleroderma renal crisis during the conditioning phase, attributed to a combination of rapid fluid and electrolyte shifts and high-dose glucocorticoids given as prophylaxis for ATG-induced cytokine storm. In some children with JIA, a fatal macrophage activation syndrome occurred, thought to be an infection triggered by the profound immunosuppression resulting from TBI and CD34 purging (19). These toxicity problems were mostly eliminated by lung shielding, concurrent ACE inhibition, and reduced intensity of the regimen, respectively. However, an inevitable TRM will always exist, which must be weighed against the potential long-term benefit, a calculation that requires efficacy data from the randomized trials.

Late complications include not only the well-known fungal and other opportunistic infections during the T-cell reconstitution phase (which may last up to 2 years or more) but also the emergence of second autoimmunity (20). It is almost always antigen specific (e.g., platelet, erythrocyte, thyroid) and often, but not always, resolves as the Treg network is reconstituted. However, some patients have succumbed to this (e.g., acquired hemophilia A antibodies after HSCT for MS) (21).

\section{NONRESPONSE AND RELAPSE}

Two-thirds of the transplanted patients did not respond or responded and then relapsed. The factors determining this remain elusive, but some studies in RA suggested that clinical responders $(n=5)$ had a larger number of cells at baseline expressing CD3, CD4, CD27, CD45RA, CD45RB, and CD45RO in synovium $(P<0.05)$, higher activity on human immunoglobulin $\mathrm{G}(\mathrm{HIg})$ scans $(P=0.08)$, and a trend toward higher concentrations of C-reactive protein (CRP) in serum than nonresponders $(n=2)$. Subsequent remissions and relapses in responders paralleled reduction and reexpression, respectively, of T-cell markers. A relatively increased expression of $\mathrm{CD} 45 \mathrm{RB}$ and $\mathrm{CD} 45 \mathrm{RO}$ on synovial $\mathrm{CD} 3^{+} \mathrm{T}$ cells was seen after high-dose chemotherapy + autologous stem cell transplantation. No correlations were found between disease activity score and changes in B cells or macrophage infiltration or synoviocytes (22).

\section{PEDIATRIC EXPERIENCE}

Although JIA in most children may be controlled by conventional drugs, including anti-interleukin-1 and -6 biologics, some children do not respond. For such children, autologous HSCT has been successfully performed since 1997 . The longterm outcome of the initial cohort of children with resistant JIA, treated with HSCT, has recently been summarized (23). The initial cohort of children was treated with a conditioning regimen containing CYC, ATGs and low-dose TBI. Overall, favorable responses were seen, with a drug-free remission rate of $50-55 \%$. In the more recent years, late relapses up to 7 years after HSCT have been recorded. The observed relapses were often less severe compared with the situation before HSCT and could be treated successfully with conventional drugs in most cases. More recently, autologous stem cell transplantation was performed in four JIA children with a fludarabine-containing regimen instead of low-dose TBI. With a 4- to 5-year follow-up, these four patients are all in drug-free full remission.

The same group has recently also reported on a successful outcome of autologous HSCT in two cases of therapy refractory juvenile dermatomyositis. Both patients had developed contractures and were wheelchair dependent despite therapy including methotrexate, steroids, immunoglobulins, cyclosporin A, and rituximab. HSCT was performed using a CD3/CD19-depleted graft after immunoablative conditioning with fludarabine, CYC, and ATG. This induced a dramatic improvement and sustained remission of the disease in both patients (24)

\section{SUMMARY OF AUTOLOGOUS HSCT FOR AD}

Autologous HSCT for severe AD had demonstrated remarkable clinical, laboratory, and morphological improvement in many patients, but at a high price, including a TRM up to $10 \%$ in some conditions. Retrospective analysis from established databases is inevitably incomplete, especially in those patients "lost to follow-up" and assumed to be still alive and/ or in drug-free remission. The advent of the biologics has reduced the need for more radical therapies such as HSCT in RA and MS, but for SSc and severe forms of Crohn's disease it remains an option. The results of prospective, randomized clinical trials will be critical in deciding the future of this treatment. 


\section{MESENCHYMAL STEM CELL TRANSPLANTATION FOR AD}

Mesenchymal stem cells are stromally derived adult progenitor cells, more accurately called multipotent mesenchymal stromal cells (MSCs), because their true "stemness" has not been established. They may be derived from various tissues including bone marrow, placenta and umbilical cord, fat, and teeth. Although they comprise a heterogeneous group of progenitor cells, they have been defined by consensus as being plastic adherent, bearing certain stromal surface markers (CD76, CD90, and CD105), and lacking hematopoietic cell markers such as CD11a, CD14, CD19, CD34, CD45, and MHC class II. In addition, they should have at least adipogenic, osteogenic, and chondrogenic differentiation potential (25).

First applied in humans for hematopoietic stem cell graft enhancement over 15 years ago (26), there has since developed a major interest in their potential for immune-modulating, anti-inflammatory, and tissue protective properties, including $\mathrm{AD}$ (27). Originally considered "regenerative" because of their ability to transdifferentiate into other tissues, it is now appreciated that the positive effects seen in vitro, in animal models, and in some clinical studies are most likely caused by their capacity to initiate various paracrine events, resulting in tissue protection. Some of these effects are via soluble factors such as transforming growth factor- $\beta, 2,3$-indoleamine dioxygenase, soluble human leukocyte antigen- $G$, and others via cell-cell contact and "reprogramming" of target cells (28). In addition, MSCs seem to display certain special properties such as immune privilege (survival in allogeneic environments) and active homing to distressed tissues via surface molecules such as CXCR4. They also preferentially home to tumor stroma (29), potentially inhibiting tumor immune surveillance.

The role of MSCs in normal tissue homeostasis and repair is not fully understood, but it seems likely that they participate in the inflammatory niche, possibly as part of the resolution phase of injury. Their origin in adult animals is also not defined fully, but potential sources are pericytes released from blood vessels during injury (30), epidermal to mesenchymal transition, especially in the lung and kidney (31), and direct release from the bone marrow. In any case, the use of supraphysiological numbers of exogenous MSCs in vivo may evoke different biological pathways from those employed during homeostasis.

\section{CLINICAL EXPERIENCE}

Following many positive animal models of inflammation, organ transplant, autoimmunity, critical ischemia, radiation damage, and tissue scarring, MSCs entered clinical trials for inflammatory disorders, first in graft-vs.-host disease, then later in MS, Crohn's disease (including fistula closure), SLE, and SSc (reviewed in ref. 27). In addition, many trials relating to ischemia in the myocardium, central nervous system, kidney, and limbs have been performed.

Despite this activity, only 14 case reports or small phase I/ II clinical trial in $\mathrm{AD}$ have been published (Table 2), all of $<15$ patients up to the time of writing (October 2011). Two large randomized, prospective trials in Crohn's disease and graft-vs.-host disease were reported as failing to reach their primary endpoints, but as yet have not been published in the peer-reviewed literature.

There is a lack of standardization of cell product regarding heterogeneity, potency, impact of expansion media on phenotype, and suitability of the source. Many expansion media employ growth factors such as fibroblast growth factor- $\beta$, which has been shown to induce proliferation-dependent major histocompatibility complex class II expression (32) and, in one study, suspected karyotypic changes (33). The

Table 2. Phase I/II clinical trials of MSC in autoimmune disease

\begin{tabular}{lrlll}
\hline Autoimmune disease & Patient no. & MSC product & Route & Outcome \\
\hline Multiple sclerosis & 10 & Allogeneic bone marrow & Intrathecal & Mixed \\
Multiple sclerosis & 10 & Autologous bone marrow & IVI & Improvement? MRI-no impact \\
Multiple sclerosis & 15 & Autologous bone marrow & Intrathecal (all) + IVI (5) & Some stabilized \\
Multiple sclerosis & 3 & Mixed allo/auto fat & Mixed IVI + intrathecal & Improved clinic MRI—no impact \\
Multiple sclerosis & 1 & Allogeneic umbilical cord & IVI & 42 \\
Crohn's fistulae & 14 & Autologous fat & Intra-fistula & Improved \\
Crohn's fistulae & 10 & Autologous bone marrow & Intra-fistula & $71 \%$ fistula closure \\
Crohn's fistulae & 10 & Autologous bone marrow & IVI & $70 \%$ full closure 30\% partial closure \\
Scleroderma digital ulcer & 2 & Autologous blood and marrow MNC & Intralesional & 45 \\
Scleroderma & 1 & Allogeneic bone marrow & IVI & Some improved \\
SLE nephritis & 15 & Allogeneic bone marrow & IVI & 46 \\
SLE nephritis & 16 & Allogeneic umbilical cord & IVI & Improved \\
SLE nephritis & 2 & Autologous bone marrow & IVI & Improved \\
SLE-lung hemorrhage & 1 & Allogeneic umbilical cord & IVI & Improved \\
DM type 2 & 10 & Allogeneic placenta & IVI $\times 3$ & No change \\
SSc limb ischemia & 1 & Autologous bone marrow & IVI & Improved \\
& & & & 48 \\
\hline
\end{tabular}

DM, diabetes mellitus; IVI, intravenous infusion; MNC, mononuclear cell; MRI, magnetic resonance imaging; MSC, mesenchymal stem cell; SLE, systemic lupus erythematosus; SSc, systemic sclerosis. 
clinical trials so far have used MSCs derived from various sources including fat, bone marrow, placenta, and umbilical cord, with the former two being either autologous or allogeneic.

MSCs from the bone marrow of AD patients have been shown to be defective regarding certain functions such as differentiation potential and hematopoietic support, but seem equally potent as healthy allogeneic MSCs in terms of in vitro antiproliferative potential (34).

The efficacy data so far available are difficult to interpret because of variable pre-MSC transplant treatment regimens, nonstandardized outcome measures, and lack of long-term follow-up.

So far, no acute toxicity signals have emerged from the experience with approximately 1,000 patients (35), although longer-term data regarding tumor surveillance are important.

\section{FUTURE DIRECTIONS}

Clearly, further small phase I/II trials will not shed further light on the long-term benefit of MSCs and we now need larger, randomized, double-blind clinical trials, including mechanistic side studies. There are major gaps in our knowledge such as duration of engraftment, impact on normal tissues and organs, and phenotypic changes occurring in the MSCs when exposed to the inflammatory/ischemic target tissue. Interferon- $\gamma$ and fibroblast growth factor- $\beta$ will both cause expression of major histocompatibility complex class II on MSCs, and in the latter case, this molecule is able to present antigens (32).

Several groups are planning such studies: The EULAR Stromal Cell Group is finalizing a prospective, double-blind, comparative, multicenter trial of lupus nephritis using allogeneic MSCs, and an MS consortium will perform a prospective comparative trial using autologous MSCs (36).

A concentrated effort from investigators, regulators, and industries is required to determine through adequately powered, prospective clinical trials the potential benefit of MSC transplantation in autoimmune and other human disorders. Because many of these are investigator-initiated, strategy-based studies, the bureaucratic burden may hamper development (37).

\section{KEY POINTS}

- Autologous HSCT for severe AD may induce remission in some patients. The results of large randomized phase II trials are awaited to determine whether the significant toxicity is offset by durable drug-free remission.

- Such transplants should only be performed in centers with extensive experience in HSCT.

- MSCs show promise as immunomodulatory agents in ADs with minimal acute toxicity. Large randomized trials are needed to confirm this impression.

- The exact mode of action of the MSC is unclear, but most likely short-term, paracrine-mediated antiinflammatory and antiproliferative modulation of the inflammatory "niche" occur.

\section{REFERENCES}

1. Tyndall A, Gratwohl A. Blood and marrow stem cell transplants in autoimmune disease. A consensus report written on behalf of the European League Against Rheumatism (EULAR) and the European Group for Blood and Marrow Transplantation (EBMT). Br J Rheumatol 1997;36:390-2.

2. Jacobs P, Vincent MD, Martell RW. Prolonged remission of severe refractory rheumatoid arthritis following allogeneic bone marrow transplantation for drug-induced aplastic anaemia. Bone Marrow Transplant 1986;1:237-9.

3. Knaan-Shanzer S, Houben P, Kinwel-Bohré EP, van Bekkum DW. Remission induction of adjuvant arthritis in rats by total body irradiation and autologous bone marrow transplantation. Bone Marrow Transplant 1991;8:333-8.

4. Tamm M, Gratwohl A, Tichelli A, Perruchoud AP, Tyndall A. Autologous haemopoietic stem cell transplantation in a patient with severe pulmonary hypertension complicating connective tissue disease. Ann Rheum Dis 1996;55:779-80.

5. Tyndall A, Black C, Finke J, et al. Treatment of systemic sclerosis with autologous haemopoietic stem cell transplantation. Lancet 1997;349:254.

6. Tyndall A, Gratwohl A. Blood and marrow stem cell transplants in autoimmune disease: a consensus report written on behalf of the European League against Rheumatism (EULAR) and the European Group for Blood and Marrow Transplantation (EBMT). Bone Marrow Transplant 1997;19:643-5.

7. Gratwohl A, Passweg J, Bocelli-Tyndall C, et al.; Autoimmune Diseases Working Party of the European Group for Blood and Marrow Transplantation (EBMT). Autologous hematopoietic stem cell transplantation for autoimmune diseases. Bone Marrow Transplant 2005;35:869-79.

8. Alexander T, Thiel A, Rosen O, et al. Depletion of autoreactive immunologic memory followed by autologous hematopoietic stem cell transplantation in patients with refractory SLE induces long-term remission through de novo generation of a juvenile and tolerant immune system. Blood 2009;113:214-23.

9. Muraro PA, Douek DC, Packer A, et al. Thymic output generates a new and diverse TCR repertoire after autologous stem cell transplantation in multiple sclerosis patients. J Exp Med 2005;201:805-16.

10. van Wijk F, Roord ST, Vastert B, de Kleer I, Wulffraat N, Prakken BJ. Regulatory $\mathrm{T}$ cells in autologous stem cell transplantation for autoimmune disease. Autoimmunity 2008;41:585-91.

11. Verrecchia F, Laboureau J, Verola O, et al. Skin involvement in scleroderma-where histological and clinical scores meet. Rheumatology (Oxford) 2007;46:833-41.

12. Fleming JN, Nash RA, McLeod DO, et al. Capillary regeneration in scleroderma: stem cell therapy reverses phenotype? PLoS ONE 2008;3:e1452.

13. Aschwanden M, Halter JP, Walker UA, et al. Nail fold capillaroscopy differs widely between systemic sclerosis and chronic graft vs host disease of the skin. Rheumatology (Oxford) 2011;50:1168-9.

14. Couzin-Frankel J. Immunology. Replacing an immune system gone haywire. Science 2010;327:772-4.

15. Burt RK, Shah SJ, Dill K, et al. Autologous non-myeloablative haemopoietic stem-cell transplantation compared with pulse cyclophosphamide once per month for systemic sclerosis (ASSIST): an open-label, randomised phase 2 trial. Lancet 2011;378:498-506.

16. Tyndall A. Stem cells: HSCT for systemic sclerosis-swallows and summers. Nat Rev Rheumatol 2011;7:624-6.

17. van Laar JM, Farge D, Tyndall A. Autologous Stem cell Transplantation International Scleroderma (ASTIS) trial: hope on the horizon for patients with severe systemic sclerosis. Ann Rheum Dis 2005;64:1515.

18. Bartoloni E, Shoenfeld Y, Gerli R. Inflammatory and autoimmune mechanisms in the induction of atherosclerotic damage in systemic rheumatic diseases: two faces of the same coin. Arthritis Care Res (Hoboken) 2011;63:178-83.

19. Brinkman DM, de Kleer IM, ten Cate R, et al. Autologous stem cell transplantation in children with severe progressive systemic or polyarticular juvenile idiopathic arthritis: long-term follow-up of a prospective clinical trial. Arthritis Rheum 2007;56:2410-21. 
20. Daikeler T, Tzankov A, Hoenger G, et al. Minimal T-cell requirements for triggering haemophagocytosis associated with Epstein-Barr virus-driven B-cell proliferation: a clinical case study. Ann Rheum Dis 2011;70:1338-9.

21. Kaloyannidis P, Sakellari I, Fassas A, et al. Acquired hemophilia-A in a patient with multiple sclerosis treated with autologous hematopoietic stem cell transplantation and interferon beta-1a. Bone Marrow Transplant 2004;34:187-8.

22. Verburg RJ, Flierman R, Sont JK, et al. Outcome of intensive immunosuppression and autologous stem cell transplantation in patients with severe rheumatoid arthritis is associated with the composition of synovial T cell infiltration. Ann Rheum Dis 2005;64:1397-405.

23. Wulffraat NM, van Rooijen EM, Tewarie R, Brinkman D, Prakken B, Kuis W. Current perspectives of autologous stem cell transplantation for severe Juvenile Idiopathic Arthritis. Autoimmunity 2008;41:632-8.

24. Holzer U, van Royen-Kerkhof A, van der Torre P, et al. Successful autologous stem cell transplantation in two patients with juvenile dermatomyositis. Scand J Rheumatol 2010;39:88-92.

25. Horwitz EM, Le Blanc K, Dominici M, et al.; International Society for Cellular Therapy. Clarification of the nomenclature for MSC: The International Society for Cellular Therapy position statement. Cytotherapy 2005;7:393-5.

26. Lazarus HM, Koc ON, Devine SM, et al. Cotransplantation of HLA-identical sibling culture-expanded mesenchymal stem cells and hematopoietic stem cells in hematologic malignancy patients. Biol Blood Marrow Transplant 2005;11:389-98.

27. Tyndall A, Uccelli A. Multipotent mesenchymal stromal cells for autoimmune diseases: teaching new dogs old tricks. Bone Marrow Transplant 2009;43:821-8.

28. Németh K, Leelahavanichkul A, Yuen PS, et al. Bone marrow stromal cells attenuate sepsis via prostaglandin $\mathrm{E}(2)$-dependent reprogramming of host macrophages to increase their interleukin-10 production. Nat Med 2009;15:42-9.

29. Dwyer RM, Potter-Beirne SM, Harrington KA, et al. Monocyte chemotactic protein-1 secreted by primary breast tumors stimulates migration of mesenchymal stem cells. Clin Cancer Res 2007;13:5020-7.

30. Caplan AI. All MSCs are pericytes? Cell Stem Cell 2008;3:229-30.

31. Kalluri R. EMT: when epithelial cells decide to become mesenchymal-like cells. J Clin Invest 2009;119:1417-9.

32. Bocelli-Tyndall C, Zajac P, Di Maggio N, et al. Fibroblast growth factor 2 and platelet-derived growth factor, but not platelet lysate, induce proliferation-dependent, functional class II major histocompatibility complex antigen in human mesenchymal stem cells. Arthritis Rheum 2010;62:3815-25.

33. Tarte K, Gaillard J, Lataillade JJ, et al.; Société Française de Greffe de Moelle et Thérapie Cellulaire. Clinical-grade production of human mesenchymal stromal cells: occurrence of aneuploidy without transformation. Blood 2010;115:1549-53.

34. Bocelli-Tyndall C, Bracci L, Spagnoli G, et al. Bone marrow mesenchymal stromal cells (BM-MSCs) from healthy donors and auto-immune disease patients reduce the proliferation of autologous- and allogeneic-stimulated lymphocytes in vitro. Rheumatology (Oxford) 2007;46:403-8.

35. Martin I, Baldomero H, Bocelli-Tyndall C, Slaper-Cortenbach I, Passweg J, Tyndall A. The survey on cellular and engineered tissue therapies in Europe in 2009. Tissue engineering Part A 2011;17:2221-30.

36. Freedman MS, Bar-Or A, Atkins HL, et al.; MSCT Study Group. The therapeutic potential of mesenchymal stem cell transplantation as a treatment for multiple sclerosis: consensus report of the International MSCT Study Group. Mult Scler 2010;16:503-10.

37. Tyndall A. Why do we need noncommercial, investigator-initiated clinical trials? Nat Clin Pract Rheumatol 2008;4:354-5.

38. Aschwanden M, Daikeler T, Jaeger KA, et al. Rapid improvement of nailfold capillaroscopy after intense immunosuppression for systemic sclerosis and mixed connective tissue disease. Ann Rheum Dis 2008;67:1057-9.

39. Mohyeddin Bonab M, Yazdanbakhsh S, Lotfi J, et al. Does mesenchymal stem cell therapy help multiple sclerosis patients? Report of a pilot study. Iran J Immunol 2007;4:50-7.

40. Yamout B, Hourani R, Salti H, et al. Bone marrow mesenchymal stem cell transplantation in patients with multiple sclerosis: a pilot study. J Neuroimmunol 2010;227:185-9.

41. Karussis D, Karageorgiou C, Vaknin-Dembinsky A, et al. Safety and immunological effects of mesenchymal stem cell transplantation in patients with multiple sclerosis and amyotrophic lateral sclerosis. Arch Neurol 2010;67:1187-94.

42. Riordan NH, Ichim TE, Min WP, et al. Non-expanded adipose stromal vascular fraction cell therapy for multiple sclerosis. J Transl Med 2009;7:29.

43. Liang J, Zhang H, Hua B, et al. Allogeneic mesenchymal stem cells transplantation in treatment of multiple sclerosis. Mult Scler 2009;15: 644-6.

44. Garcia-Olmo D, Herreros D, Pascual I, et al. Expanded adipose-derived stem cells for the treatment of complex perianal fistula: a phase II clinical trial. Dis Colon Rectum 2009;52:79-86.

45. Ciccocioppo R, Bernardo ME, Sgarella A, et al. Autologous bone marrowderived mesenchymal stromal cells in the treatment of fistulising Crohn's disease. Gut 2011;60:788-98.

46. Duijvestein M, Vos AC, Roelofs $\mathrm{H}$, et al. Autologous bone marrow-derived mesenchymal stromal cell treatment for refractory luminal Crohn's disease: results of a phase I study. Gut 2010;59:1662-9.

47. Nevskaya T, Ananieva L, Bykovskaia S, et al. Autologous progenitor cell implantation as a novel therapeutic intervention for ischaemic digits in systemic sclerosis. Rheumatology (Oxford) 2009;48:61-4.

48. Christopeit M, Schendel M, Föll J, Müller LP, Keysser G, Behre G. Marked improvement of severe progressive systemic sclerosis after transplantation of mesenchymal stem cells from an allogeneic haploidentical-related donor mediated by ligation of CD137L. Leukemia 2008;22:1062-4.

49. Liang J, Zhang H, Hua B, et al. Allogenic mesenchymal stem cells transplantation in refractory systemic lupus erythematosus: a pilot clinical study. Ann Rheum Dis 2010;69:1423-9.

50. Sun L, Wang D, Liang J, et al. Umbilical cord mesenchymal stem cell transplantation in severe and refractory systemic lupus erythematosus. Arthritis Rheum 2010;62:2467-75.

51. Carrion F, Nova E, Ruiz C, et al. Autologous mesenchymal stem cell treatment increased $\mathrm{T}$ regulatory cells with no effect on disease activity in two systemic lupus erythematosus patients. Lupus 2010;19:317-22.

52. Liang J, Gu F, Wang H, et al. Mesenchymal stem cell transplantation for diffuse alveolar hemorrhage in SLE. Nat Rev Rheumatol 2010;6:486-9.

53. Jiang R, Han Z, Zhuo G, et al. Transplantation of placenta-derived mesenchymal stem cells in type 2 diabetes: a pilot study. Front Med 2011;5:94-100.

54. Guiducci S, Porta F, Saccardi R, et al. Autologous mesenchymal stem cells foster revascularization of ischemic limbs in systemic sclerosis: a case report. Ann Intern Med 2010;153:650-4. 